\title{
Axiomatizing the Skew Boolean Propositional Calculus
}

\author{
R. Veroff \\ University of New Mexico
}

M. Spinks

La Trobe University

April 14, 2007

\begin{abstract}
The skew Boolean propositional calculus $(S B P C)$ is a generalization of the classical propositional calculus that arises naturally in the study of certain well-known deductive systems. In this article, we consider a candidate presentation of $S B P C$ and prove it constitutes a Hilbert-style axiomatization. The problem reduces to establishing that the logic presented by the candidate axiomatization is algebraizable in the sense of Blok and Pigozzi. In turn, this is equivalent to verifying four particular formulas are derivable from the candidate presentation. Automated deduction methods played a central role in proving these four theorems. In particular, our approach relied heavily on the method of proof sketches.
\end{abstract}

\section{Introduction}

With increasing frequency, mathematicians are approaching members of the automated deduction community for help solving problems in their own research areas. These mathematicians generally don't care what systems we use - Otter[15], Prover9[17] or Waldmeister[10], for example - and they don't care what additional procedures we employ. They just want their problems solved. In this article, we describe our solution to a problem - a set of four theorems to prove - that we found to be especially challenging. Finding these four proofs involved the use of some fairly tedious side procedures, but automated deduction methods played the central and most critical role.

The four theorems are in a system called Skew Boolean Propositional Calculus $(S B P C)$, which is a "noncommutative" analog of (the negation-free fragment of) classical propositional calculus. The theorems together establish the algebraizability of $S B P C$, a result that is fundamental to the study of "pointed discriminator logics" presented in [3]. The four theorems are difficult, presumably too difficult to establish by hand, and it's not at all obvious if it's possible to establish them by other semantic means (such as using Kripke semantics). At the least, establishing them by semantic means would require the development of suitable semantic methods (completeness theorems and the like).

(c) 2007 Kluwer Academic Publishers. Printed in the Netherlands. 
The remainder of this article consists of a problem statement and a description of our approach to finding the proofs. A single proof that combines the four results into one is presented in the appendix.

\section{Problem Statement}

It is well known that $C P C$, the classical propositional calculus, is determined by the collection of 2-element truth tables:

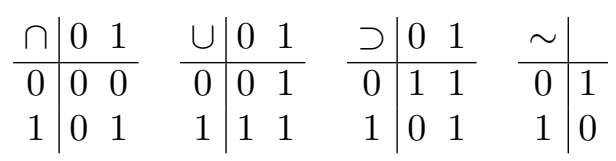

with 1 being the designated value. Formally, $C P C$ is the deductive system determined by the logical matrix $\langle\mathbf{2},\{1\}\rangle$, where $\mathbf{2}$ denotes the 2-element Boolean algebra.

The connectives $\cap, \cup$ and $\supset$ admit a number of generalizations to the $n$-valued case in the literature. Several of these, first described in [2], are as follows. For each $1 \leq n \in \omega$, let $M_{n}:=\{0,1, \ldots, n\}$. Consider the operations $\wedge, \vee, \Rightarrow$ and $\rightarrow$ defined for all $a, b \in M_{n}$ by:

$$
\begin{aligned}
& a \wedge b:=\left\{\begin{array}{l}
b \text { if } a=1 \\
a \text { otherwise, }
\end{array} \quad a \vee b:= \begin{cases}1 \text { if } a=1 \\
b \text { otherwise }\end{cases} \right. \\
& a \Rightarrow b:=\left\{\begin{array}{ll}
1 & \text { if } a=b \\
b & \text { otherwise, }
\end{array} \quad a \rightarrow b:=\left\{\begin{array}{l}
1 \text { if } a \neq 1 \\
b \text { otherwise }
\end{array}\right.\right.
\end{aligned}
$$

The operations $\wedge, \vee, \Rightarrow$ and $\rightarrow$ are called conjunction, disjunction and strong and weak implication, respectively. The implication $a \Rightarrow b$ is strong in the sense that the relation $\leq$ defined for all $a, b \in M_{n}$ by $a \leq b$ if and only if $a \Rightarrow b=1$ is a partial ordering on $A$. In contrast, $a \rightarrow b$ is weak in the sense that the relation $\preceq$ defined for all $a, b \in M_{n}$ by $a \preceq b$ if and only if $a \rightarrow b=1$ is only a quasiordering (reflexive and transitive relation) on $A$.

For each $1 \leq n \in \omega$, let $\mathbf{M}_{n}$ denote the algebra $\left\langle M_{n} ; \wedge, \vee, \Rightarrow, \rightarrow, 1\right\rangle$ and $\mathcal{M}_{n}$ the logical matrix $\left\langle\mathbf{M}_{n},\{1\}\right\rangle$. Observe that, when $n=1$, $a \wedge b=a \cap b, a \vee b=a \cup b$, and $a \Rightarrow b=a \supset b=a \rightarrow b$ for all $a, b \in M_{1}$. Hence, in the two-valued case, the logic determined by the matrix $\mathcal{M}_{1}$ is $C P C^{\wedge, \vee}, \supset$, the negation-free fragment of the classical propositional calculus (equivalently, the axiomatic extension of Hilbert's positive logic [18] by the Peirce law). In contrast, when $n \geq 2$, each matrix $\mathcal{M}_{n}$ determines an $n+1$-valued logic. For example, the 3 -valued logic 
determined by $\mathcal{M}_{2}$ has truth tables

\begin{tabular}{|c|c|c|c|c|c|c|c|}
\hline & $\begin{array}{lll}0 & 1 & 2\end{array}$ & & $\begin{array}{llll}0 & 1 & 2\end{array}$ & $\Rightarrow$ & $\begin{array}{lll}0 & 12\end{array}$ & & $\left|\begin{array}{ll}0 & 1\end{array}\right|$ \\
\hline 0 & $\begin{array}{lll}0 & 0 & 0\end{array}$ & 0 & $\begin{array}{lll}0 & 1\end{array}$ & 0 & 112 & 0 & 11 \\
\hline 1 & $\begin{array}{lll}0 & 1 & 2\end{array}$ & 1 & 11 & 1 & 01 & 1 & $\begin{array}{ll}0 & 1\end{array}$ \\
\hline ? & $\begin{array}{lll}2 & 2\end{array}$ & 2 & $\begin{array}{lll}0 & 1\end{array}$ & 2 & 01 & 2 & 11 \\
\hline
\end{tabular}

and from inspection of these tables, it is easy to see that $(0 \rightarrow 2) \rightarrow$ $(0 \Rightarrow 2)=2 \neq 1$, whence $\mathcal{M}_{2}$ is not $C P C^{\wedge, \vee, \supset}$. It follows that no $\mathcal{M}_{n}$, with $n \geq 2$, is $C P C^{\wedge, \vee, \supset}$ either, since in each case $\mathcal{M}_{2}$ is a submatrix of $\mathcal{M}_{n}$.

For each $1 \leq n<\omega$, the logic determined by $\mathcal{M}_{n}$ is called the $n+1$ valued skew Boolean propositional calculus. The logic determined by the countable-valued matrix $\mathcal{M}_{\omega}$, in symbols $S B P C$, is called simply the skew Boolean propositional calculus.

It turns out that $S B P C$ arises naturally in the study of non-classical deductive systems. In more detail, the (ternary) discriminator on a set $A$ is the function $t: A^{3} \rightarrow A$ defined for all $a, b, c \in A$ by

$$
t(a, b, c):= \begin{cases}c & \text { if } a=b \\ a & \text { otherwise. }\end{cases}
$$

An equationally definable class of algebras $\mathrm{V}$ is said to be a discriminator variety if it is generated by a class of discriminator algebras. A pointed discriminator variety is a discriminator variety having a constant term. For a study of the discriminator in universal algebra, see Burris and Sankappanavar [6, Chapter 4].

Recall next that a deductive system $S$ over a language type $\Lambda$ is said to be algebraizable in the sense of Blok and Pigozzi [4] if there exists a quasi-equationally definable class of algebras $\mathrm{K}$, having the same language type as $S$, such that the $S$-consequence relation $\vdash_{S}$ and the equational consequence relation $\models_{\mathrm{K}}$ are interpretable in one another in a certain strong sense. The class $\mathrm{K}$ is called the equivalent quasivariety semantics of $S$. Informally, $\mathrm{K}$ is an equivalent quasivariety semantics for $S$ if it stands in relation to $S$ just as the class of Boolean algebras stands in relation to the classical propositional calculus.

A deductive system $S$ is called a pointed discriminator logic if it is algebraizable and its equivalent quasivariety semantics is a pointed discriminator variety. Examples of such logics abound in the literature and include: the classical propositional calculus; the normal modal logic S5; the basic fuzzy logics with Baaz delta $[9,1,24]$; the $n$-dimensional cylindric logics; the $n$-valued Post logics; the $n$-valued Łukasiewicz logics; and the tetravalent modal logic of Font and Rius [8, 13].

In [3] it is shown that every pointed discriminator logic arises (up to definitional equivalence) as an axiomatic expansion of $S B P C$ by ex- 
tensional logical connectives. (For a complementary study of axiomatic expansions of fragments of the intuitionistic propositional calculus, see Czelakowski and Pigozzi [7].) For example, it is shown in [3] that $\mathbf{S 5}$ is an axiomatic expansion of $S B P C$ by the logical connectives $\cap, \cup, \supset, \sim$ of the classical propositional calculus; the other pointed discriminator logics described above can be analogously presented. SBPC may therefore be understood as the deductive system "inherent" in (or common to) any pointed discriminator logic. It is this observation that motivates our interest in and study of $S B P C$.

Consider now the following collection of axioms $A x$ and inference rules $I r$ over the language type of $S B P C$, comprising nine axioms taken from the presentation of the "pointed fixedpoint discriminator" logic $B C S K$ given in $[11,12,19,20]^{1}$ :

$$
\begin{aligned}
& x \Rightarrow(y \Rightarrow x) \\
& (x \Rightarrow(y \Rightarrow z)) \Rightarrow((x \Rightarrow y) \Rightarrow(x \Rightarrow z)) \\
& ((x \Rightarrow y) \Rightarrow x) \Rightarrow x \\
& x \Rightarrow(y \rightarrow x) \\
& (x \rightarrow(y \rightarrow z)) \Rightarrow((x \rightarrow y) \rightarrow(x \rightarrow z)) \\
& (x \rightarrow(y \rightarrow z)) \Rightarrow(y \rightarrow(x \rightarrow z)) \\
& ((x \rightarrow y) \rightarrow x) \Rightarrow x \\
& ((x \Rightarrow y) \rightarrow y) \Rightarrow((y \Rightarrow x) \rightarrow x) \\
& (x \Rightarrow y) \rightarrow(x \rightarrow y)
\end{aligned}
$$

together with the following six axioms for $\wedge$ and $\vee$ :

$$
\begin{aligned}
& x \rightarrow(x \vee y) \\
& y \Rightarrow(x \vee y) \\
& (x \rightarrow z) \rightarrow((y \rightarrow z) \rightarrow((x \vee y) \rightarrow z)) \\
& (x \wedge y) \Rightarrow x \\
& (x \wedge y) \rightarrow y \\
& (x \Rightarrow y) \Rightarrow((x \Rightarrow z) \Rightarrow(x \Rightarrow(y \wedge z)))
\end{aligned}
$$

and the rule of inference:

$$
x, x \rightarrow y \vdash y .
$$

$\left(\mathrm{MP}_{\rightarrow}\right)$

1 The "pointed fixedpoint discriminator" logic BCSK has traditionally been presented by the set of axioms (A1)-(A9) together with the rule of inference $(\mathrm{MP} \rightarrow)$. Axiom $(\mathrm{A} 6)$ is known to be dependent in this presentation [25], and we note that (A6) plays no role in the proof of the four desired theses given in the appendix. 
Note that modus ponens for $\Rightarrow$, in symbols $\left(\mathrm{MP}_{\Rightarrow}\right)$, follows from (A9) and two applications of $\left(\mathrm{MP}_{\rightarrow}\right)$.

Let $C$ (for "candidate") denote the deductive system axiomatized by $A x \cup \operatorname{Ir}$. By results of [3], $C P C^{\wedge, \vee, \supset}$ is the axiomatic extension of $C$ by the axiom

$$
(x \rightarrow y) \Rightarrow(x \Rightarrow y)
$$

on the one hand, while $B C S K$ is the $\{\Rightarrow, \rightarrow\}$-fragment of $C$ on the other.

Our goal was to show that the collection of axioms and inference rules $A x \cup \operatorname{Ir}$ constitutes an axiomatization of $S B P C$ (or equivalently, that $C$ is $S B P C$ ). From general results of algebraic logic, it follows that $A x \cup I r$ is a presentation of $S B P C$ if and only if $C$ is algebraizable in the sense of Blok and Pigozzi. It turns out that this is very convenient from the perspective of the work reported in [3], since the algebraizability of $S B P C$ is the foundation on which the edifice of [3] rests. Among the various possible approaches for showing that $A x \cup \operatorname{Ir}$ constitutes a complete axiomatization, therefore, our preferred method was to verify the algebraizability of $C$.

One of the central results of [4] describes a set of purely syntactic conditions that are necessary and sufficient for a deductive system to be algebraizable. In particular, these conditions assert that $C$ is algebraizable if and only if the formulas

$$
\begin{aligned}
& (\varphi \Rightarrow \psi) \rightarrow((\varphi \vee \chi) \Rightarrow(\psi \vee \chi)) \\
& (\varphi \Rightarrow \psi) \rightarrow((\chi \vee \varphi) \Rightarrow(\chi \vee \psi)) \\
& (\varphi \Rightarrow \psi) \rightarrow((\psi \Rightarrow \varphi) \rightarrow((\varphi \wedge \chi) \Rightarrow(\psi \wedge \chi))) \\
& (\varphi \Rightarrow \psi) \rightarrow((\chi \wedge \varphi) \Rightarrow(\chi \wedge \psi))
\end{aligned}
$$

are theses of $C .^{2}$

The challenge for automated reasoning was to show that (1)-(4) are indeed syntactic consequences of $A x \cup I r$, together with the derived rule of inference $\left(\mathrm{MP}_{\Rightarrow}\right) .^{3}$

\footnotetext{
2 This statement implicitly assumes the algebraizability of $B C S K$, a result which is now part of the folklore of pointed discriminator logics. By Blok and Pigozzi's syntactic conditions for algebraizability, $B C S K$ is algebraizable if and only if the formulas $(\varphi \Rightarrow \psi) \rightarrow((\chi \rightarrow \varphi) \Rightarrow(\chi \rightarrow \psi))$ and $(\varphi \Rightarrow \psi) \rightarrow((\psi \rightarrow \chi) \Rightarrow(\varphi \rightarrow \chi))$ are derivable from $(\mathrm{A} 1)-(\mathrm{A} 9)$ and $(\mathrm{MP} \rightarrow)$. If the algebraizability of $B C S K$ is not assumed, then six theses must be derived to verify the algebraizability of $C$ : the four formulas (1)-(4) together with the two formulas of this footnote.

${ }^{3}$ It is natural to ask why the four desired theses were simply not appended to the candidate axiomatization of $C$ to provide a presentation that is vacuously complete. The reason lies in the fact that one of the recurring themes of [3] is the extent
} 


\section{Finding the Proofs}

Problems in $C$ are easily represented for a resolution theorem prover. If we let $\mathrm{P}(\mathrm{t})$ represent the assertion that $\mathrm{t}$ is a theorem, then applications of $\left(\mathrm{MP}_{\rightarrow}\right)$ and $\left(\mathrm{MP}_{\Rightarrow}\right)$ can be implemented by using hyperresolution and the following clauses.

$$
\begin{array}{l|l|l}
-P(x->y) & -P(x) & P(y) . \\
-P(x=>y) & -P(x) & P(y) .
\end{array}
$$

Our search for proofs of the four challenge theorems relied very heavily on the method of proof sketches [22]. The basic idea is to find a proof of a simplified version of the theorem with relaxed constraints and then to systematically refine and transform the proof into a syntactic proof of the original. In this case, we relied on a general approach that has proven to be effective for numerous logic problems having similar syntactic structures (for example, including applications of modus ponens). This approach can be summarized with the following four high-level steps.

1. Prove an algebraic form of the theorem that includes term-level equality substitution (paramodulation) as an inference rule.

2. Reprove the algebraic form of the theorem, relying strictly on resolution and the explicit use of equality substitution axioms.

3. Reprove the theorem in its original logical form, but still include equalities and term-level substitutions.

4. Systematically eliminate all references to equality in the problem statement and proofs.

Before describing each of the four steps in more detail (specifically, in the context of the four challenge theorems), we give a brief introduction to the method of proof sketches.

\section{The Method of Proof Sketches}

Much of our work in proving difficult theorems involves sequences of Otter and Prover9 experiments and relies heavily on the use of hints [21] and on the method of proof sketches [22]. Under the hints strategy, a

$\overline{\text { to which }} S B P C$ resembles $C P C$. In particular, the candidate axiomatization of $S B P C$ mirrors one of the standard presentations of classical propositional logic (see for instance [5]). Adjoining the four theses as axioms would destroy the symmetry between $A x \cup I r$ and this standard presentation. 
generated clause is given special consideration (as defined by the user) if it subsumes ${ }^{4}$ or is subsumed by a user-supplied hint clause. The hints strategy is closely related to the weighting strategy [14], in which clauses are assigned weights that are used to help direct the search for a proof. In contrast to weighting, the hints strategy focuses directly on the identification of key clauses rather than on the general calculation of weights. Any generated clause that subsumes or is subsumed by a user-supplied hint clause is identified as being "interesting". The weight of such a clause is adjusted (either positively or negatively) according to user preferences; the cases of subsuming a hint, being subsumed by a hint, or both are controlled separately. Being based on subsumption, the hints strategy adds a semantic or logical component to the evaluation of a clause.

A proof sketch for a theorem $T$ is a sequence of clauses giving a set of conditions sufficient to prove $T$. In the ideal case, a proof sketch consists of a sequence of lemmas, where each lemma is fairly easy to prove. In any case, the clauses of a proof sketch identify potentially notable milestones on the way to finding a proof. From a strategic standpoint, it is desirable to recognize when we have achieved such milestones and to adapt the continued search for a proof accordingly. In particular, we wish to focus our attention on such milestone results and pursue their consequences sooner rather than later.

The hints strategy provides a natural and effective way to take full advantage of a proof sketch in the search for a proof. Including each clause from the proof sketch as a hint clause and making an Otter assignment such as

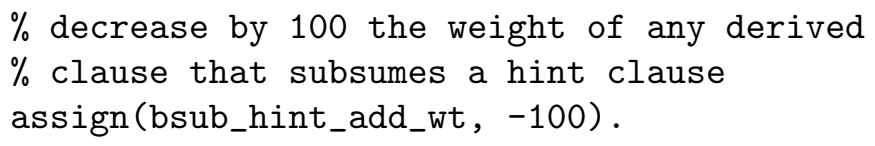

virtually ensures that when a clause is derived that subsumes a hint clause - in particular, one of the key milestone clauses of a proof sketch - the newly generated clause will become the focus of attention (that is, chosen as the "given" clause) as soon as possible.

The use of hints is additive in the sense that hints from multiple proof sketches or from sketches for different parts of a proof can all be included at the same time. For this reason, hints are particularly valuable for "gluing" subproofs together and completing partial proofs, even when wildly different search strategies were used to find the individual subproofs.

\footnotetext{
4 Subsumption normally includes deletion of subsumed clauses. Here we use the term simply as a convenient way to refer to the subsumption relationship between clauses.
} 
In [22], we consider how the generation and use of proof sketches, together with the sophisticated strategies and procedures supported by an automated reasoning program such as Otter, can be used to find proofs to challenging theorems, including open questions. The general approach is to find proofs with additional assumptions and then to systematically eliminate these assumptions from the input set, using all previous proofs as hints.

We now return to a description of the steps we took to prove the four challenge theorems in the candidate system $C$.

Step 1. Prove an algebraic form of the theorem that includes term-level equality substitution (paramodulation) as an inference rule.

In order to permit term-level substitutions, every axiom $t$ of $C$ was represented with the algebraic formula $t=1$, a procedure justified by algebraizability (see [7, Section 1.5]), and paramodulation was permitted as an inference rule. For example, axiom (A1) became

$$
\mathrm{x}=>(\mathrm{y} \Rightarrow \mathrm{x})=1 .
$$

and the two modus ponens rules, $\left(\mathrm{MP}_{\rightarrow}\right)$ and $\left(\mathrm{MP}_{\Rightarrow}\right)$, became

$$
\begin{aligned}
& \mathrm{x} !=1|\mathrm{x} \rightarrow \mathrm{y} !=1| \mathrm{y}=1 \text {. } \\
& \mathrm{x} !=1|\mathrm{x} \Rightarrow \mathrm{y} !=1| \mathrm{y}=1 \text {. }
\end{aligned}
$$

respectively. We included, in addition, the following clauses.

$$
\begin{aligned}
& \mathrm{x} \rightarrow \mathrm{y} \quad !=1 \quad \mid \mathrm{y} \rightarrow \mathrm{x} \quad !=1 \quad \mathrm{x}=\mathrm{y} \text {. } \\
& \mathrm{x} \Rightarrow \mathrm{y} !=1|\mathrm{y} \Rightarrow \mathrm{x} !=1| \mathrm{x}=\mathrm{y} \text {. }
\end{aligned}
$$

Adding the two preceding clauses is equivalent algebraically to adding axiom (A10) in its algebraic form $(x \rightarrow y) \Rightarrow(x \Rightarrow y)=1$. The equational theory in which we are working at this point is thus that of the equivalent algebraic semantics of $C P C^{\wedge, \vee, \supset}$, namely, the class of generalized Boolean algebras.

Finding these proofs was not especially difficult, but the searches relied on the method of proof sketches. The intermediate steps made use of several additional assumptions, for example, a set of identities relevant to the larger work presented in [3].

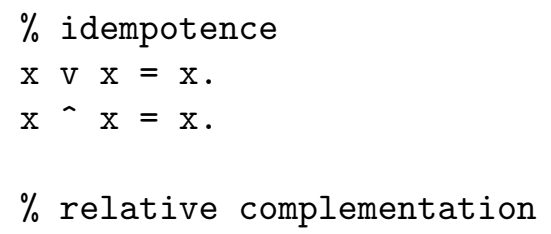




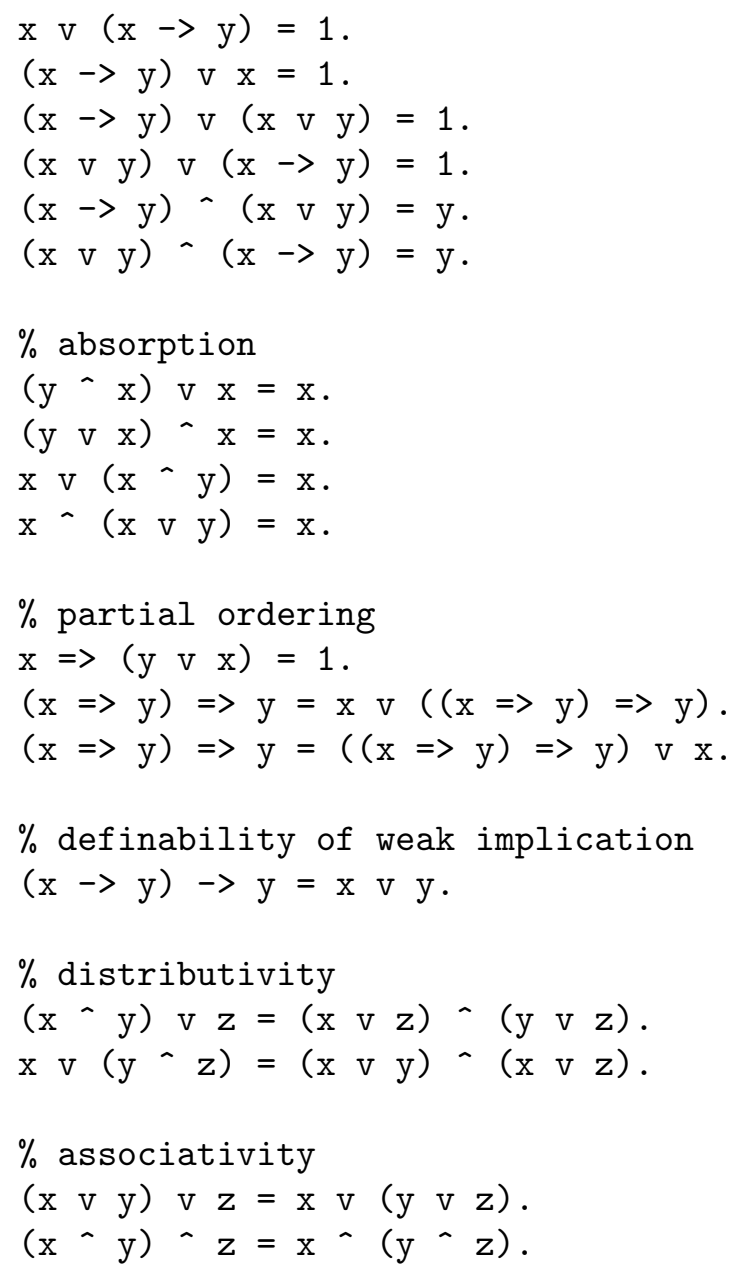

We also included as initial extra assumptions the following demodulators, all of which are known to follow from the identities above.

$$
\begin{aligned}
& x \rightarrow 1=1 . \\
& 1->x=x . \\
& x \Rightarrow 1=1 . \\
& 1 \Rightarrow x=x .
\end{aligned}
$$

All of the additional assumptions were then systematically eliminated in a sequence of proofs.

We stress that the term-level substitutions we relied on in this step are not all sound in $C$; what we're doing here is more than simply transforming the syntactic structure of a proof. The method of proof sketches focuses on the generation of sufficient conditions for proving a 
theorem, conditions that in turn are used to provide strategic guidance for following proof searches. We have ample empirical evidence of the effectiveness of the basic approach. See, for example, [23] and [16].

Step 2. Reprove the algebraic form of the theorem, relying strictly on resolution and the explicit use of equality substitution axioms.

It is known that every paramodulation step in a proof can be replaced by a sequence of resolution steps with equality axioms such as the following.

$$
\begin{array}{ll|l}
\mathrm{x} & !=\mathrm{y} & \mathrm{x} \rightarrow \mathrm{z}=\mathrm{y} \rightarrow \mathrm{z} . \\
\mathrm{x} & !=\mathrm{y} \mid \mathrm{z} \rightarrow \mathrm{x}=\mathrm{z} \rightarrow \mathrm{y} .
\end{array}
$$

The intention here was to provide all of the intermediate resolvents as hints for future proofs.

In theory, we could have written special procedures to generate all of these resolvents, but we elected to use Otter to find them for us. This turned out to be significantly more difficult (and tedious) than we anticipated, even when focusing on individual paramodulation steps. Several of these required multiple Otter runs with carefully selected input clauses and processing parameters. We also ended up writing scripts to help automate some of the editing. ${ }^{5}$

Step 3. Reprove the theorem in its original logical form, but still include equalities and term-level substitutions.

There are no equalities in the original statement of the problem, but they were introduced in this step by first including the clauses,

$$
\begin{array}{l|l|l}
-P(x->y) & -P(y->x) & x=y \\
-P(x=>y) & -P(y=>x) & x=y
\end{array}
$$

and eventually-with proof sketches-reducing this to

$$
-P(x=y) \quad|-P(y=x)| x=y \text {. }
$$

The equality substitution axioms used in previous steps remained, and we added

$$
\mathrm{x} !=\mathrm{y}|-\mathrm{P}(\mathrm{x})| \mathrm{P}(\mathrm{y}) \text {. }
$$

for substitutions into the predicate P.

In order to take full advantage of all of the proof sketches we had previously accumulated, it was necessary to translate the hint clauses as well. In particular, every hint clause of the form

\footnotetext{
${ }^{5}$ We believe we have convinced Bill McCune to include the option of generating such expanded proofs automatically in a future release of Prover9.
} 
$t=1$.

was replaced by

$P(t)$.

It is easy to see that, in the underlying (quasi-) equational theory, $t_{1}=t_{2}$ if and only if $t_{1} \Rightarrow t_{2}=1$ and $t_{2} \Rightarrow t_{1}=1$, and that the latter two equalities imply $t_{1} \rightarrow t_{2}=1$ and $t_{2} \rightarrow t_{1}=1$, respectively. Hence, for every hint clause of the form

$$
\mathrm{t} 1=\mathrm{t} 2
$$

we included the four clauses

$$
\begin{aligned}
& \mathrm{P}(\mathrm{t} 1 \rightarrow \mathrm{t} 2) . \\
& \mathrm{P}(\mathrm{t} 2 \rightarrow \mathrm{t} 1) . \\
& \mathrm{P}(\mathrm{t} 1=\mathrm{t} 2) . \\
& \mathrm{P}(\mathrm{t} 2=>\mathrm{t} 1) .
\end{aligned}
$$

as additional hint clauses.

It may seem that the previous proof - the result of Step 2-would map directly to a proof in this representation, but this is not quite the case. The primary difficulty is that paramodulations from equalities of the form

$$
t=1 .
$$

(introducing the constant 1 into the resulting clause), do not have direct analogs in the new representation. Nevertheless, with some effort (and several Otter runs), we eventually found the desired proofs.

Step 4. Systematically eliminate all references to equality in the problem statement and proofs.

Recall that the proof sketches we had accumulated up to this point are not complete in that they include steps that are not theorems in $C$. There are gaps in the proofs that need to be filled in within the theory. Eliminating equality was a difficult and tedious process.

We first replaced the equality substitution axioms with P-form analogs based on equivalences, for example, including

$$
\begin{aligned}
& -P(x=>y)|-P(y=x)|-P\left(\begin{array}{llllll}
x & v & z
\end{array}\right) \mid P(y \quad v \quad z) . \\
& -P(x=y)|-P(y=x)|-P(z \vee x) \mid P(z \vee y) \text {. }
\end{aligned}
$$


for operator $\vee$. We also included transitivity laws for $\rightarrow$ and $\Rightarrow$, which are provable in $C$.

$$
\begin{array}{l|l|l|l}
-P(x=>y) & -P(y=>z) & P(x=>z) . \\
-P(x \rightarrow y) & -P(y->z) & P(x \rightarrow z) .
\end{array}
$$

Proving this version of the theorems was difficult, requiring several runs and the generation of several intermediate proof sketches. The intermediate assumptions we used included numerous P-form analogs based on equivalences of the term-level equality substitution axioms. These were restricted to the first few levels of nesting, for example,

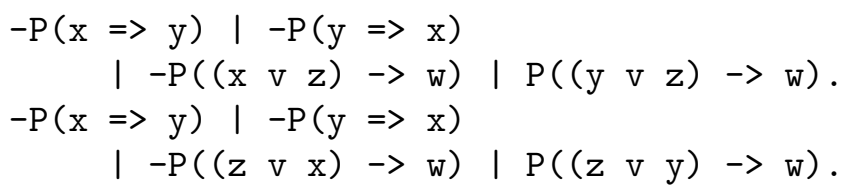

Finally, we systematically eliminated all of the extra assumptionsthat is, all clauses not appearing in the original problem statementuntil we had the sought-after proofs of the four theorems. This was not especially difficult from a strategic standpoint, but it was tedious, since we were able to eliminate only a very few extra assumptions at a time. We found that if we eliminated too many at once, it was too difficult to find the next proof in the sequence.

\section{Final Comments}

We were able to use hints and sketches in a systematic way to prove what we believe to be very difficult theorems. Some of the steps, however, were mind bogglingly tedious rather than being mathematically or strategically interesting. The good news is that we believe much of this can be automated. We already rely on various editor macros, shell scripts, programs and special modifications to Otter to help with the manipulation of clauses and input files, and these have helped tremendously. We're currently developing an autosketches mode for Prover9 that will handle some of the iterative aspects of the method of proof sketches.

From a mathematical perspective, the axiomatization $A x \cup \operatorname{Ir}$ of $S B P C$ provided in this work is germane for concrete applications of the main result of [3] - that is, for presenting arbitrary pointed discriminator logics as axiomatic expansions of $S B P C$. The point is that Otter has not only played an important role in proving several difficult theorems; in helping to verify that $S B P C$ is algebraizable, it has 
actively contributed toward the nascent development of a nontrivial mathematical theory. 


\section{Appendix}

Here, combined into a single proof, are the derivations of the four challenge theorems (1) through (4). These appear, respectively, at steps 228, 224, 248 and 241. The justification for each deduction is a triple consisting of the inference rule $\left(\mathrm{MP}_{\rightarrow}\right.$ or $\left.\mathrm{MP}_{\Rightarrow}\right)$, the major premise, and then the minor premise.

1. $x \Rightarrow(y \Rightarrow x)$

$[\mathrm{A} 1]$

2. $(x \Rightarrow(y \Rightarrow z)) \Rightarrow((x \Rightarrow y) \Rightarrow(x \Rightarrow z))$

$[\mathrm{A} 2]$

3. $((x \Rightarrow y) \Rightarrow x) \Rightarrow x$

[A3]

4. $x \Rightarrow(y \rightarrow x)$

[A4]

5. $(x \rightarrow(y \rightarrow z)) \Rightarrow((x \rightarrow y) \rightarrow(x \rightarrow z))$

6. $((x \rightarrow y) \rightarrow x) \Rightarrow x$

7. $((x \Rightarrow y) \rightarrow y) \Rightarrow((y \Rightarrow x) \rightarrow x)$

8. $(x \Rightarrow y) \rightarrow(x \rightarrow y)$

9. $x \rightarrow(x \vee y)$

[A11]

10. $x \Rightarrow(y \vee x)$

[A12]

11. $(x \rightarrow y) \rightarrow((z \rightarrow y) \rightarrow((x \vee z) \rightarrow y))$

[A13]

12. $(x \wedge y) \Rightarrow x$

13. $(x \wedge y) \rightarrow y$

14. $(x \Rightarrow y) \Rightarrow((x \Rightarrow z) \Rightarrow(x \Rightarrow(y \wedge z)))$

[A16]

15. $x \Rightarrow(y \Rightarrow(z \Rightarrow y))$

16. $((x \Rightarrow(y \Rightarrow z)) \Rightarrow(x \Rightarrow y)) \Rightarrow((x \Rightarrow(y \Rightarrow z))$

$[\mathrm{MP} \Rightarrow, 1,1]$ $\Rightarrow(x \Rightarrow z))$

$\left[\mathrm{MP}_{\Rightarrow}, 2,2\right]$

17. $x \Rightarrow((y \Rightarrow(z \Rightarrow u)) \Rightarrow((y \Rightarrow z) \Rightarrow(y \Rightarrow u)))$

$\left[\mathrm{MP}_{\Rightarrow}, 1,2\right]$

18. $(x \Rightarrow y) \Rightarrow(x \Rightarrow x)$

$\left[\mathrm{MP}_{\Rightarrow}, 2,1\right]$

19. $x \Rightarrow(((y \Rightarrow z) \Rightarrow y) \Rightarrow y)$

$\left[\mathrm{MP}_{\Rightarrow}, 1,3\right]$

20. $x \rightarrow(y \Rightarrow(z \rightarrow y))$

$\left[\mathrm{MP}_{\Rightarrow}, 4,4\right]$

21. $x \Rightarrow(y \Rightarrow(z \rightarrow y))$

$[\mathrm{MP} \Rightarrow, 1,4]$

22. $x \rightarrow(y \Rightarrow(z \Rightarrow y))$

$[\mathrm{MP} \Rightarrow, 4,1]$

23. $x \Rightarrow((y \rightarrow(z \rightarrow u)) \Rightarrow((y \rightarrow z) \rightarrow(y \rightarrow u)))$

$\left[\mathrm{MP}_{\Rightarrow}, 1,5\right]$

24. $((x \Rightarrow y) \rightarrow y) \rightarrow((y \Rightarrow x) \rightarrow x)$

$\left[\mathrm{MP}_{\rightarrow}, 8,7\right]$

25. $((x \rightarrow y) \rightarrow x) \rightarrow x$

$\left[\mathrm{MP}_{\rightarrow}, 8,6\right]$

26. $x \rightarrow(y \rightarrow x)$

$\left[\mathrm{MP}_{\rightarrow}, 8,4\right]$

27. $(x \Rightarrow(y \Rightarrow z)) \rightarrow((x \Rightarrow y) \Rightarrow(x \Rightarrow z))$

$\left[\mathrm{MP}_{\rightarrow}, 8,2\right]$

28. $x \rightarrow(y \Rightarrow x)$

29. $x \rightarrow((y \Rightarrow z) \rightarrow(y \rightarrow z))$

$\left[\mathrm{MP}_{\rightarrow}, 8,1\right]$

30. $x \rightarrow(y \rightarrow(y \vee z))$

$\left[\mathrm{MP}_{\Rightarrow}, 4,8\right]$

31. $x \rightarrow(y \Rightarrow(z \vee y))$

$\left[\mathrm{MP}_{\Rightarrow}, 4,9\right]$

32. $x \Rightarrow(y \Rightarrow(z \vee y))$

$\left[\mathrm{MP}_{\Rightarrow}, 4,10\right]$

$[\mathrm{MP} \Rightarrow, 1,10]$ 
33. $x \Rightarrow((y \wedge z) \Rightarrow y)$

$[\mathrm{MP} \Rightarrow, 1,12]$

34. $x \rightarrow((y \wedge z) \rightarrow z)$

$\left[\mathrm{MP}_{\Rightarrow}, 4,13\right]$

35. $x \rightarrow(y \rightarrow(z \rightarrow y))$

$\left[\mathrm{MP}_{\rightarrow}, 26,26\right]$

36. $x \rightarrow(y \rightarrow(z \Rightarrow y))$

$\left[\mathrm{MP}_{\rightarrow}, 26,28\right]$

37. $(x \Rightarrow y) \Rightarrow(x \Rightarrow(z \Rightarrow y))$

$\left[\mathrm{MP}_{\Rightarrow}, 2,15\right]$

38. $x \Rightarrow x$

$\left[\mathrm{MP}_{\Rightarrow}, 18,15\right]$

39. $x \rightarrow x$

$\left[\mathrm{MP}_{\rightarrow}, 8,38\right]$

40. $(x \Rightarrow y) \Rightarrow(x \Rightarrow(x \wedge y))$

$\left[\mathrm{MP}_{\Rightarrow}, 14,38\right]$

41. $(x \rightarrow y) \rightarrow((y \vee x) \rightarrow y)$

$\left[\mathrm{MP}_{\rightarrow}, 11,39\right]$

42. $(x \Rightarrow y) \Rightarrow(x \Rightarrow(z \rightarrow y))$

$\left[\mathrm{MP}_{\Rightarrow}, 2,21\right]$

43. $(x \rightarrow y) \rightarrow(x \rightarrow(y \vee z))$

$\left[\mathrm{MP}_{\Rightarrow}, 5,30\right]$

44. $(x \Rightarrow y) \Rightarrow(x \Rightarrow(z \vee y))$

$\left[\mathrm{MP}_{\Rightarrow}, 2,32\right]$

45. $(x \Rightarrow(y \wedge z)) \Rightarrow(x \Rightarrow y)$

$\left[\mathrm{MP}_{\Rightarrow}, 2,33\right.$

46. $(x \rightarrow(y \wedge z)) \rightarrow(x \rightarrow z)$

$\left[\mathrm{MP}_{\Rightarrow}, 5,34\right]$

47. $(x \rightarrow y) \rightarrow(x \rightarrow(z \Rightarrow y))$

$\left[\mathrm{MP}_{\Rightarrow}, 5,36\right]$

48. $(x \Rightarrow((y \Rightarrow z) \Rightarrow y)) \Rightarrow(x \Rightarrow y)$

$\left[\mathrm{MP}_{\Rightarrow}, 2,19\right]$

49. $(x \rightarrow(y \Rightarrow z)) \rightarrow(x \rightarrow(y \rightarrow z))$

$[\mathrm{MP} \Rightarrow, 5,29]$

50. $(x \Rightarrow y) \rightarrow(x \Rightarrow(z \Rightarrow y))$

$\left[\mathrm{MP}_{\rightarrow}, 8,37\right]$

51. $(x \Rightarrow y) \rightarrow(x \Rightarrow(z \rightarrow y))$

$\left[\mathrm{MP}_{\rightarrow}, 8,42\right]$

52. $(x \rightarrow(y \rightarrow z)) \Rightarrow(u \rightarrow((x \rightarrow y) \rightarrow(x \rightarrow z)))$

$\left[\mathrm{MP}_{\Rightarrow}, 42,5\right]$

53. $(x \Rightarrow y) \rightarrow(x \Rightarrow(z \vee y))$

$\left[\mathrm{MP}_{\rightarrow}, 8,44\right]$

54. $(x \Rightarrow(y \wedge z)) \Rightarrow(u \Rightarrow(x \Rightarrow y))$

$\left[\mathrm{MP}_{\Rightarrow}, 37,45\right]$

55. $x \rightarrow(y \Rightarrow(x \vee z))$

56. $(((x \Rightarrow y) \rightarrow y) \rightarrow(y \Rightarrow x)) \rightarrow(((x \Rightarrow y) \rightarrow y)$

$\left[\mathrm{MP}_{\rightarrow}, 47,9\right]$ $\rightarrow x)$

$\left[\mathrm{MP}_{\Rightarrow}, 5,24\right]$

57. $((x \Rightarrow y) \Rightarrow(x \wedge z)) \Rightarrow x$

58. $(((x \rightarrow y) \Rightarrow z) \Rightarrow y) \Rightarrow(x \rightarrow y)$

$\left[\mathrm{MP}_{\Rightarrow}, 48,45\right]$

59. $((x \Rightarrow y) \Rightarrow(x \wedge z)) \rightarrow x$

$\left[\mathrm{MP}_{\Rightarrow}, 48,42\right]$

$\left[\mathrm{MP}_{\rightarrow}, 8,57\right]$

60. $(((x \rightarrow y) \Rightarrow z) \Rightarrow y) \rightarrow(x \rightarrow y)$

$\left[\mathrm{MP}_{\rightarrow}, 8,58\right]$

61. $((((x \rightarrow y) \Rightarrow z) \Rightarrow y) \rightarrow x) \rightarrow(((x \rightarrow y) \Rightarrow z)$

$$
\Rightarrow y) \rightarrow y)
$$

$\left[\mathrm{MP}_{\Rightarrow}, 5,60\right]$

62. $(x \Rightarrow(y \Rightarrow(z \Rightarrow u))) \Rightarrow(x \Rightarrow((y \Rightarrow z) \Rightarrow(y$ $\Rightarrow u))$

$\left[\mathrm{MP}_{\rightarrow}, 27,17\right]$

63. $(x \Rightarrow(y \rightarrow(z \rightarrow u))) \Rightarrow(x \Rightarrow((y \rightarrow z) \rightarrow(y$ $\rightarrow u))$

$\left[\mathrm{MP}_{\rightarrow}, 27,23\right]$

$\left[\mathrm{MP}_{\rightarrow}, 56,31\right]$

$\left[\mathrm{MP}_{\rightarrow}, 56,22\right]$

$\left[\mathrm{MP}_{\rightarrow}, 56,20\right]$

$\left[\mathrm{MP}_{\Rightarrow}, 62,1\right]$

$\left[\mathrm{MP}_{\rightarrow}, 8,67\right]$

68. $(x \Rightarrow y) \rightarrow((z \Rightarrow x) \Rightarrow(z \Rightarrow y))$

$[\mathrm{MP} \Rightarrow, 67,48]$

64. $(((x \vee y) \Rightarrow y) \rightarrow y) \rightarrow(x \vee y)$

66. $(((x \rightarrow y) \Rightarrow y) \rightarrow y) \rightarrow(x \rightarrow y)$

66. $(((x \rightarrow y) \Rightarrow y) \rightarrow y) \rightarrow(x \rightarrow y)$ 
70. $(x \Rightarrow y) \rightarrow((z \Rightarrow x) \rightarrow(z \Rightarrow y))$

71. $(x \rightarrow(y \rightarrow z)) \Rightarrow((u \rightarrow(x \rightarrow y)) \rightarrow(u \rightarrow(x$

$\left[\mathrm{MP}_{\rightarrow}, 49,68\right]$ $\rightarrow z))$

$\left[\mathrm{MP}_{\Rightarrow}, 63,52\right]$

72. $(x \rightarrow y) \Rightarrow((z \rightarrow x) \rightarrow(z \rightarrow y))$

$\left[\mathrm{MP}_{\Rightarrow}, 63,4\right]$

73. $(x \rightarrow y) \rightarrow((z \rightarrow x) \rightarrow(z \rightarrow y))$

$\left[\mathrm{MP}_{\rightarrow}, 8,72\right]$

74. $(x \rightarrow(y \Rightarrow z)) \rightarrow(x \rightarrow(y \Rightarrow(u \Rightarrow z)))$

$\left[\mathrm{MP}_{\Rightarrow}, 72,50\right]$

75. $(x \rightarrow((y \rightarrow z) \rightarrow y)) \rightarrow(x \rightarrow y)$

$\left[\mathrm{MP}_{\Rightarrow}, 72,25\right]$

76. $(((x \Rightarrow y) \rightarrow z) \rightarrow y) \rightarrow(x \Rightarrow y)$

$\left[\mathrm{MP}_{\rightarrow}, 75,47\right]$

77. $(x \rightarrow(((y \Rightarrow z) \rightarrow u) \rightarrow z)) \rightarrow(x \rightarrow(y \Rightarrow z))$

$\left[\mathrm{MP}_{\rightarrow}, 73,76\right]$

78. $((x \wedge y) \Rightarrow(x \Rightarrow z)) \Rightarrow((x \wedge y) \Rightarrow z)$

$\left[\mathrm{MP}_{\Rightarrow}, 16,33\right]$

79. $((((x \rightarrow(y \rightarrow x)) \rightarrow z) \Rightarrow u) \Rightarrow z) \rightarrow z$

$\left[\mathrm{MP}_{\rightarrow}, 61,35\right]$

80. $((x \rightarrow(y \rightarrow x)) \rightarrow z) \Rightarrow z$

$\left[\mathrm{MP}_{\rightarrow}, 65,79\right]$

81. $(x \Rightarrow((y \rightarrow(z \rightarrow y)) \rightarrow u)) \Rightarrow(x \Rightarrow u)$

$\left[\mathrm{MP}_{\rightarrow}, 68,80\right]$

82. $(x \rightarrow(y \rightarrow z)) \Rightarrow(y \rightarrow(x \rightarrow z))$

$[\mathrm{MP} \Rightarrow, 81,71]$

83. $(x \rightarrow y) \rightarrow((y \rightarrow z) \rightarrow(x \rightarrow z))$

$\left[\mathrm{MP}_{\Rightarrow}, 82,73\right]$

84. $(x \Rightarrow y) \rightarrow((y \Rightarrow z) \rightarrow(x \Rightarrow z))$

$\left[\mathrm{MP}_{\Rightarrow}, 82,70\right]$

85. $x \rightarrow((x \rightarrow(y \wedge z)) \rightarrow z)$

86. $(x \vee y) \rightarrow((y \rightarrow x) \rightarrow x)$

$\left[\mathrm{MP}_{\Rightarrow}, 82,46\right]$

$\left[\mathrm{MP}_{\Rightarrow}, 82,41\right]$

87. $x \rightarrow((x \rightarrow y) \rightarrow y)$

88. $(x \rightarrow y) \rightarrow((z \rightarrow y) \rightarrow((z \vee x) \rightarrow y))$

$\left[\mathrm{MP}_{\Rightarrow}, 82,39\right]$

$\left[\mathrm{MP}_{\Rightarrow}, 82,11\right]$

$[\mathrm{MP} \Rightarrow, 82,8]$

89. $x \rightarrow((x \Rightarrow y) \rightarrow y)$

$\mathrm{MP}_{\rightarrow}, 87,54$

90. $(((x \Rightarrow(y \wedge z)) \Rightarrow(u \Rightarrow(x \Rightarrow y))) \rightarrow v) \rightarrow v$
91. $(((x \rightarrow y) \rightarrow(z \rightarrow y)) \rightarrow u) \rightarrow((z \rightarrow x) \rightarrow u)$

$\left[\mathrm{MP}_{\rightarrow}, 83,83\right]$

92. $(x \rightarrow(y \Rightarrow(x \rightarrow z))) \rightarrow(y \Rightarrow(x \rightarrow z))$

$\left[\mathrm{MP}_{\rightarrow}, 77,83\right]$

93. $(((x \rightarrow y) \rightarrow y) \rightarrow z) \rightarrow(x \rightarrow z)$

$\left[\mathrm{MP}_{\rightarrow}, 83,87\right]$

94. $((x \Rightarrow(y \rightarrow z)) \rightarrow u) \rightarrow((x \Rightarrow z) \rightarrow u)$

$\left[\mathrm{MP}_{\rightarrow}, 83,51\right.$

95. $((x \rightarrow y) \rightarrow z) \rightarrow((x \Rightarrow y) \rightarrow z)$

96. $((x \Rightarrow(y \Rightarrow z)) \Rightarrow u) \rightarrow((x \Rightarrow z) \Rightarrow u)$

$\left[\mathrm{MP}_{\rightarrow}, 83,8\right]$

97. $((x \rightarrow y) \Rightarrow z) \rightarrow(y \Rightarrow z)$

$\left[\mathrm{MP}_{\rightarrow}, 84,37\right]$

98. $((x \Rightarrow y) \Rightarrow z) \rightarrow(y \Rightarrow z)$

$\left[\mathrm{MP}_{\rightarrow}, 84,4\right]$

$\left[\mathrm{MP}_{\rightarrow}, 84,1\right]$

99. $(x \rightarrow((y \Rightarrow z) \Rightarrow u)) \rightarrow(x \rightarrow(z \Rightarrow u))$

$\left[\mathrm{MP}_{\rightarrow}, 73,98\right]$

100. $x \Rightarrow(y \Rightarrow(y \wedge x))$

101. $(x \Rightarrow y) \Rightarrow((x \Rightarrow(y \Rightarrow z)) \Rightarrow(x \Rightarrow z))$

$\left[\mathrm{MP}_{\rightarrow}, 98,40\right]$

$\left[\mathrm{MP}_{\rightarrow}, 98,16\right]$

102. $(x \Rightarrow y) \rightarrow(x \rightarrow(y \vee z))$

103. $(x \rightarrow(y \Rightarrow(z \vee u))) \rightarrow((x \vee(y \Rightarrow u))$

$\left[\mathrm{MP}_{\rightarrow}, 95,43\right]$

$$
\rightarrow(y \Rightarrow(z \vee u)))
$$

$\left[\mathrm{MP}_{\rightarrow}, 88,53\right]$

104. $(x \rightarrow y) \rightarrow((x \vee y) \rightarrow y)$

$\left[\mathrm{MP}_{\rightarrow}, 88,39\right]$

105. $(((x \vee y) \rightarrow y) \rightarrow z) \rightarrow((x \rightarrow y) \rightarrow z)$

$\left[\mathrm{MP}_{\rightarrow}, 83,104\right]$

106. $(x \rightarrow((y \Rightarrow(z \Rightarrow u)) \Rightarrow v)) \rightarrow(x \rightarrow((y \Rightarrow u)$

$$
\Rightarrow v) \text { ) }
$$

$\left[\mathrm{MP}_{\rightarrow}, 73,96\right]$

107. $(x \Rightarrow(y \Rightarrow z)) \rightarrow(y \Rightarrow(x \Rightarrow z))$

$\left[\mathrm{MP}_{\rightarrow}, 99,27\right]$ 


\begin{tabular}{|c|c|}
\hline 108. $(x \rightarrow(y \Rightarrow(z \Rightarrow u))) \rightarrow(x \rightarrow(z \Rightarrow(y \Rightarrow u)))$ & {$\left[\mathrm{MP}_{\rightarrow}, 73,107\right]$} \\
\hline 109. $(x \Rightarrow(y \Rightarrow z)) \rightarrow(y \rightarrow(x \Rightarrow z))$ & {$\left[\mathrm{MP}_{\rightarrow}, 49,107\right]$} \\
\hline 110. $(x \Rightarrow y) \Rightarrow((y \Rightarrow z) \Rightarrow(x \Rightarrow z))$ & {$\left[\mathrm{MP}_{\rightarrow}, 107,67\right]$} \\
\hline 111. $x \rightarrow((x \Rightarrow y) \Rightarrow(z \rightarrow y))$ & {$\left[\mathrm{MP}_{\rightarrow}, 109,42\right]$} \\
\hline 112. $x \rightarrow((x \Rightarrow y) \Rightarrow y)$ & {$\left[\mathrm{MP}_{\rightarrow}, 109,38\right]$} \\
\hline 113. $(x \Rightarrow y) \Rightarrow(x \rightarrow y)$ & {$\left[\mathrm{MP}_{\rightarrow}, 92,111\right]$} \\
\hline 114. $((x \rightarrow y) \Rightarrow z) \rightarrow((x \Rightarrow y) \Rightarrow z)$ & {$\left[\mathrm{MP}_{\rightarrow}, 84,113\right]$} \\
\hline 115. $(x \Rightarrow(y \Rightarrow z)) \rightarrow(x \Rightarrow(y \rightarrow z))$ & {$\left[\mathrm{MP}_{\rightarrow}, 70,113\right]$} \\
\hline 116. $(((x \Rightarrow y) \Rightarrow(z \Rightarrow y)) \Rightarrow u) \Rightarrow((z \Rightarrow x) \Rightarrow u)$ & {$\left[\mathrm{MP}_{\Rightarrow}, 110,110\right]$} \\
\hline 117. $((x \Rightarrow y) \Rightarrow z) \Rightarrow((z \Rightarrow x) \Rightarrow x)$ & {$\left[\mathrm{MP}_{\Rightarrow}, 69,110\right]$} \\
\hline 118. $x \Rightarrow(y \rightarrow(y \wedge x))$ & {$\left[\mathrm{MP}_{\rightarrow}, 115,100\right]$} \\
\hline 119. $(x \Rightarrow(y \Rightarrow z)) \Rightarrow((x \Rightarrow y) \rightarrow(x \Rightarrow z))$ & {$\left[\mathrm{MP}_{\rightarrow}, 115,2\right]$} \\
\hline 120. $((x \Rightarrow(y \rightarrow(y \wedge x))) \Rightarrow z) \rightarrow z$ & {$\left[\mathrm{MP}_{\rightarrow}, 89,118\right]$} \\
\hline 121. $(x \Rightarrow((y \Rightarrow z) \Rightarrow u)) \rightarrow(x \Rightarrow((u \Rightarrow y) \Rightarrow y))$ & {$\left[\mathrm{MP}_{\rightarrow}, 70,117\right]$} \\
\hline 122. $((x \Rightarrow y) \Rightarrow z) \rightarrow((z \Rightarrow x) \Rightarrow x)$ & {$\left[\mathrm{MP}_{\rightarrow}, 8,117\right]$} \\
\hline 123. $x \Rightarrow((y \Rightarrow(x \Rightarrow z)) \Rightarrow(y \Rightarrow z))$ & {$\left[\mathrm{MP}_{\rightarrow}, 98,101\right]$} \\
\hline 124. $(x \Rightarrow(y \Rightarrow z)) \Rightarrow(y \Rightarrow(x \Rightarrow z))$ & {$\left[\mathrm{MP}_{\rightarrow}, 107,123\right]$} \\
\hline 125. $((x \Rightarrow(y \Rightarrow z)) \Rightarrow u) \Rightarrow((y \Rightarrow(x \Rightarrow z)) \Rightarrow u)$ & {$\left[\mathrm{MP}_{\Rightarrow}, 110,124\right]$} \\
\hline 126. $\begin{array}{c}(x \Rightarrow(y \Rightarrow(z \Rightarrow u))) \\
\Rightarrow u)))\end{array}$ & {$\left[\mathrm{MP}_{\rightarrow}, 68,119\right]$} \\
\hline 127. $(((x \Rightarrow y) \rightarrow z) \rightarrow u) \rightarrow((u \rightarrow y) \rightarrow(x \Rightarrow y))$ & {$\left[\mathrm{MP}_{\rightarrow}, 91,77\right]$} \\
\hline 128. $(x \Rightarrow((y \Rightarrow z) \Rightarrow u)) \rightarrow((u \Rightarrow y) \Rightarrow(x \Rightarrow y))$ & {$\left[\mathrm{MP}_{\rightarrow}, 108,121\right]$} \\
\hline 129. $(x \Rightarrow((x \wedge y) \Rightarrow z)) \Rightarrow((x \wedge y) \Rightarrow z)$ & {$\left[\mathrm{MP}_{\Rightarrow}, 125,78\right]$} \\
\hline 130. $(x \Rightarrow((x \wedge y) \Rightarrow z)) \rightarrow((x \wedge y) \Rightarrow z)$ & {$\left[\mathrm{MP}_{\rightarrow}, 8,129\right]$} \\
\hline 131. $(((x \wedge y) \Rightarrow z) \rightarrow u) \rightarrow((x \Rightarrow((x \wedge y) \Rightarrow z))$ & [MP 8 \\
\hline 132. $(x \rightarrow(y \Rightarrow z)) \rightarrow(((x \rightarrow u) \rightarrow z) \rightarrow(y \Rightarrow z))$ & {$\left[\mathrm{MP}_{\rightarrow}, 91,127\right]$} \\
\hline$((x \rightarrow y) \rightarrow y) \rightarrow((x \rightarrow y) \Rightarrow y)$ & {$\left[\mathrm{MP}_{\rightarrow}, 127,66\right]$} \\
\hline 134. $((x \vee y) \rightarrow y) \rightarrow((x \vee y) \Rightarrow y)$ & {$\left[\mathrm{MP}_{\rightarrow}, 127,64\right]$} \\
\hline 135. $(((x \Rightarrow y) \rightarrow y) \rightarrow x) \rightarrow(y \Rightarrow x)$ & {$\left[\mathrm{MP}_{\rightarrow}, 127,24\right]$} \\
\hline 136. $x \rightarrow((x \rightarrow y) \Rightarrow y)$ & {$\left[\mathrm{MP}_{\rightarrow}, 93,133\right]$} \\
\hline 137. $x \rightarrow((x \rightarrow y) \Rightarrow(z \Rightarrow y))$ & {$\left[\mathrm{MP}_{\rightarrow}, 74,136\right]$} \\
\hline 138. $(x \rightarrow y) \rightarrow((x \vee y) \Rightarrow y)$ & {$\left[\mathrm{MP}_{\rightarrow}, 105,134\right]$} \\
\hline 139. $((x \rightarrow y) \rightarrow(z \Rightarrow u)) \rightarrow((x \rightarrow u) \Rightarrow(z \Rightarrow u))$ & {$\left[\mathrm{MP}_{\rightarrow}, 132,137\right]$} \\
\hline 140. $(x \rightarrow y) \Rightarrow((x \vee y) \Rightarrow y)$ & {$\left[\mathrm{MP}_{\rightarrow}, 139,138\right]$} \\
\hline 141. $(x \rightarrow y) \Rightarrow(((x \rightarrow z) \rightarrow y) \Rightarrow y)$ & {$\left[\mathrm{MP}_{\rightarrow}, 139,136\right]$} \\
\hline 142. $(((x \Rightarrow y) \rightarrow y) \rightarrow x) \Rightarrow(y \Rightarrow x)$ & {$\left[\mathrm{MP}_{\rightarrow}, 139,135\right]$} \\
\hline 143. $(x \rightarrow y) \Rightarrow(((x \rightarrow z) \Rightarrow y) \Rightarrow y)$ & {$\left[\mathrm{MP}_{\rightarrow}, 139,112\right]$} \\
\hline 144. $(((x \Rightarrow y) \Rightarrow y) \rightarrow y) \Rightarrow(x \Rightarrow y)$ & {$\left[\mathrm{MP}_{\rightarrow}, 139,65\right]$} \\
\hline 145. $(x \Rightarrow y) \Rightarrow((x \vee y) \Rightarrow y)$ & {$\left[\mathrm{MP}_{\rightarrow}, 114,140\right]$} \\
\hline 146. $(x \vee y) \Rightarrow((x \rightarrow y) \Rightarrow y)$ & {$\left[\mathrm{MP}_{\rightarrow}, 107,140\right]$} \\
\hline
\end{tabular}


147. $(x \vee y) \Rightarrow((x \Rightarrow y) \Rightarrow y)$

148. $(x \Rightarrow(y \vee z)) \rightarrow(x \Rightarrow((y \rightarrow z) \Rightarrow z))$

149. $(((x \rightarrow y) \Rightarrow y) \Rightarrow z) \Rightarrow((x \vee y) \Rightarrow z)$

150. $((x \rightarrow y) \rightarrow(x \vee z)) \Rightarrow(x \vee z)$

151. $(x \Rightarrow(((y \Rightarrow z) \rightarrow z) \rightarrow y)) \Rightarrow(x \Rightarrow(z \Rightarrow y))$

152. $(x \Rightarrow(y \rightarrow z)) \Rightarrow((y \rightarrow x) \Rightarrow(y \rightarrow z))$

153. $(x \Rightarrow(((y \Rightarrow z) \Rightarrow z) \rightarrow z)) \Rightarrow(x \Rightarrow(y \Rightarrow z))$

154. $(x \Rightarrow(y \vee z)) \rightarrow((y \rightarrow z) \Rightarrow(x \Rightarrow z))$

155. $(x \Rightarrow(((y \rightarrow z) \Rightarrow z) \Rightarrow u)) \Rightarrow(x \Rightarrow((y \vee z)$

$$
\Rightarrow u))
$$

156. $(x \rightarrow y) \Rightarrow(x \rightarrow(x \wedge y))$

157. $(x \Rightarrow(y \Rightarrow(z \rightarrow u))) \Rightarrow(x \Rightarrow((z \rightarrow y) \Rightarrow(z$ $\rightarrow u))$

158. $(x \Rightarrow(y \vee z)) \rightarrow((y \rightarrow z) \rightarrow(x \Rightarrow z))$

159. $(x \rightarrow(((y \wedge z) \Rightarrow u) \rightarrow v)) \rightarrow(x \rightarrow((y \Rightarrow)((y$

$$
\wedge z) \Rightarrow u)) \rightarrow v))
$$

160. $(x \Rightarrow y) \Rightarrow((z \rightarrow x) \Rightarrow(z \rightarrow y))$

161. $(x \rightarrow y) \Rightarrow((y \Rightarrow z) \Rightarrow(x \rightarrow z))$

162. $(x \rightarrow y) \Rightarrow(x \rightarrow(z \vee y))$

163. $((x \rightarrow(y \vee z)) \Rightarrow u) \Rightarrow((x \rightarrow z) \Rightarrow u)$

164. $(x \rightarrow(y \rightarrow z)) \Rightarrow((y \vee z) \Rightarrow(x \rightarrow z))$

165. $(((x \rightarrow y) \Rightarrow y) \Rightarrow z) \Rightarrow(x \rightarrow z)$

166. $((x \vee y) \Rightarrow z) \Rightarrow(x \rightarrow z)$

167. $(x \Rightarrow(y \rightarrow z)) \Rightarrow(y \rightarrow(x \Rightarrow z))$

168. $(x \rightarrow y) \rightarrow((y \rightarrow z) \Rightarrow(x \rightarrow z))$

169. $(x \Rightarrow y) \rightarrow((y \rightarrow z) \Rightarrow(x \rightarrow z))$

170. $(((x \Rightarrow y) \Rightarrow y) \rightarrow z) \Rightarrow(((y \Rightarrow u) \Rightarrow x) \rightarrow z)$

171. $(x \rightarrow y) \Rightarrow(((z \Rightarrow(u \rightarrow(u \wedge z))) \Rightarrow x) \rightarrow y)$

172. $(x \rightarrow y) \Rightarrow((((z \Rightarrow(u \wedge v)) \Rightarrow(w \Rightarrow(z \Rightarrow u)))$

$$
\rightarrow x) \rightarrow y)
$$

173. $(x \rightarrow y) \Rightarrow(((x \Rightarrow z) \Rightarrow(x \wedge u)) \rightarrow y)$

174. $((x \rightarrow y) \rightarrow z) \Rightarrow(x \vee z)$

175. $((x \rightarrow y) \Rightarrow z) \Rightarrow(x \vee z)$

176. $((x \rightarrow y) \rightarrow z) \rightarrow(x \vee z)$

177. $(x \Rightarrow((y \rightarrow z) \Rightarrow u)) \Rightarrow(x \Rightarrow(y \vee u))$

178. $(x \rightarrow y) \vee(x \vee z)$

179. $((x \vee y) \rightarrow(x \rightarrow z)) \rightarrow(x \rightarrow z)$

180. $(x \rightarrow((y \rightarrow z) \rightarrow u)) \rightarrow(x \rightarrow(y \vee u))$

181. $((x \rightarrow y) \rightarrow z) \rightarrow(((x \vee u) \rightarrow(x \rightarrow y)) \rightarrow z)$

182. $((x \rightarrow(y \wedge z)) \vee z) \Rightarrow(x \rightarrow z)$

183. $(x \vee y) \Rightarrow((x \rightarrow(z \wedge y)) \rightarrow y)$
$\left[\mathrm{MP}_{\rightarrow}, 107,145\right]$

$\left[\mathrm{MP}_{\rightarrow}, 70,146\right]$

$\left[\mathrm{MP}_{\Rightarrow}, 110,146\right]$

$\left[\mathrm{MP}_{\Rightarrow}, 141,9\right]$

$\left[\mathrm{MP}_{\rightarrow}, 68,142\right]$

$\left[\mathrm{MP}_{\rightarrow}, 128,143\right]$

$\left[\mathrm{MP}_{\rightarrow}, 68,144\right]$

$\left[\mathrm{MP}_{\rightarrow}, 108,148\right]$

$[\mathrm{MP} \rightarrow, 68,149]$

$\left[\mathrm{MP}_{\rightarrow}, 120,152\right]$

$\left[\mathrm{MP}_{\rightarrow}, 68,152\right]$

$\left[\mathrm{MP}_{\rightarrow}, 49,154\right]$

$\left[\mathrm{MP}_{\rightarrow}, 73,131\right]$

$\left[\mathrm{MP}_{\Rightarrow}, 157,42\right]$

$\left[\mathrm{MP}_{\rightarrow}, 107,160\right]$

$\left[\mathrm{MP}_{\Rightarrow}, 160,10\right]$

$\left[\mathrm{MP}_{\Rightarrow}, 110,162\right]$

$\left[\mathrm{MP}_{\Rightarrow}, 155,161\right]$

$\left[\mathrm{MP}_{\Rightarrow}, 161,136\right]$

$\left[\mathrm{MP}_{\Rightarrow}, 161,9\right]$

$\left[\mathrm{MP}_{\Rightarrow}, 116,165\right]$

$\left[\mathrm{MP}_{\Rightarrow}, 167,72\right]$

$\left[\mathrm{MP}_{\rightarrow}, 95,168\right]$

$\left[\mathrm{MP}_{\rightarrow}, 168,122\right]$

$\left[\mathrm{MP}_{\rightarrow}, 168,120\right]$

$\left[\mathrm{MP}_{\rightarrow}, 168,90\right]$

$\left[\mathrm{MP}_{\rightarrow}, 168,59\right]$

$\left[\mathrm{MP}_{\Rightarrow}, 163,150\right]$

$\left[\mathrm{MP}_{\rightarrow}, 114,174\right]$

$\left[\mathrm{MP}_{\rightarrow}, 8,174\right]$

$\left[\mathrm{MP}_{\rightarrow}, 68,175\right]$

$\left[\mathrm{MP}_{\Rightarrow}, 175,174\right]$

$\left[\mathrm{MP}_{\rightarrow}, 86,178\right]$

$\left[\mathrm{MP}_{\rightarrow}, 73,176\right]$

$\left[\mathrm{MP}_{\rightarrow}, 83,179\right]$

$\left[\mathrm{MP}_{\Rightarrow}, 164,85\right]$

$[\mathrm{MP} \Rightarrow, 164,46]$ 
184. $(x \Rightarrow((y \rightarrow(z \wedge u)) \vee u)) \Rightarrow(x \Rightarrow(y \rightarrow u))$

$[\mathrm{MP} \rightarrow, 68,182]$

185. $(x \rightarrow(x \wedge y)) \Rightarrow(x \Rightarrow(x \wedge y))$

$\left[\mathrm{MP}_{\Rightarrow}, 153,173\right]$

186. $(x \Rightarrow(y \rightarrow(y \wedge z))) \rightarrow(x \Rightarrow(y \Rightarrow(y \wedge z)))$

$\left[\mathrm{MP}_{\rightarrow}, 70,185\right]$

187. $((x \rightarrow y) \Rightarrow(z \Rightarrow u)) \Rightarrow(z \Rightarrow(x \vee u))$

$[\mathrm{MP} \Rightarrow, 125,177]$

188. $(x \Rightarrow(y \vee z)) \rightarrow(y \vee(x \Rightarrow z))$

$\left[\mathrm{MP}_{\rightarrow}, 180,158\right]$

189. $(x \rightarrow(y \Rightarrow(z \vee u))) \rightarrow(x \rightarrow(z \vee(y \Rightarrow u)))$

$\left[\mathrm{MP}_{\rightarrow}, 73,188\right]$

190. $(x \Rightarrow((y \rightarrow z) \Rightarrow(u \Rightarrow v))) \Rightarrow(x \Rightarrow(u \Rightarrow(y$ $\vee v))$ )

191. $(((x \Rightarrow y) \Rightarrow y) \rightarrow x) \Rightarrow(y \Rightarrow x)$

$\left[\mathrm{MP}_{\rightarrow}, 68,187\right]$

$\left[\mathrm{MP}_{\Rightarrow}, 153,170\right]$

192. $(x \Rightarrow(((y \Rightarrow z) \Rightarrow z) \rightarrow y)) \Rightarrow(x \Rightarrow(z \Rightarrow y))$

$\left[\mathrm{MP}_{\rightarrow}, 68,191\right]$

193. $((x \rightarrow y) \rightarrow z) \rightarrow((x \vee u) \vee z)$

$\left[\mathrm{MP}_{\rightarrow}, 180,181\right]$

194. $(x \vee y) \vee((x \vee z) \Rightarrow z)$

$\left[\mathrm{MP}_{\rightarrow}, 193,138\right]$

195. $(x \rightarrow y) \Rightarrow(x \Rightarrow(x \wedge y))$

$[\mathrm{MP} \rightarrow, 186,156]$

196. $x \Rightarrow((x \rightarrow y) \Rightarrow(x \wedge y))$

$\left[\mathrm{MP}_{\rightarrow}, 107,195\right]$

197. $(x \Rightarrow(y \rightarrow z)) \Rightarrow(x \Rightarrow(y \Rightarrow(y \wedge z)))$

$\left[\mathrm{MP}_{\rightarrow}, 68,195\right]$

198. $x \Rightarrow(y \Rightarrow((y \rightarrow z) \Rightarrow(y \wedge z)))$

$\left[\mathrm{MP}_{\rightarrow}, 28,196\right]$

199. $(x \wedge y) \Rightarrow((x \rightarrow z) \Rightarrow(x \wedge z))$

200. $((x \wedge y) \Rightarrow(x \rightarrow z)) \Rightarrow((x \wedge y) \Rightarrow(x \wedge z))$

201. $(x \Rightarrow(y \rightarrow z)) \Rightarrow((x \Rightarrow y) \rightarrow(x \Rightarrow(y \wedge z)))$

$\left[\mathrm{MP}_{\Rightarrow}, 78,198\right]$

$\left[\mathrm{MP}_{\rightarrow}, 27,199\right]$

$\left[\mathrm{MP}_{\Rightarrow}, 126,197\right]$

202. $((x \rightarrow(x \wedge y)) \rightarrow y) \Rightarrow((x \rightarrow(x \wedge y)) \Rightarrow y)$

$[\mathrm{MP} \Rightarrow, 192,171]$

203. $(x \Rightarrow((y \wedge z) \Rightarrow(y \rightarrow u))) \Rightarrow(x \Rightarrow((y \wedge z)$

$$
\Rightarrow(y \wedge u)))
$$

$\left[\mathrm{MP}_{\rightarrow}, 68,200\right]$

204. $(x \Rightarrow(y \rightarrow z)) \rightarrow((x \Rightarrow y) \rightarrow(x \Rightarrow(y \wedge z)))$

$\left[\mathrm{MP}_{\rightarrow}, 8,201\right]$

205. $(x \Rightarrow((y \rightarrow(y \wedge z)) \rightarrow z))$

$$
\Rightarrow(x \Rightarrow((y \rightarrow(y \wedge z)) \Rightarrow z))
$$

$\left[\mathrm{MP}_{\rightarrow}, 68,202\right]$

206. $(x \rightarrow(y \Rightarrow(z \rightarrow u))) \rightarrow(x \rightarrow((y \Rightarrow z) \rightarrow(y$

$$
\Rightarrow(z \wedge u))))
$$

$\left[\mathrm{MP}_{\rightarrow}, 73,204\right]$

207. $(x \rightarrow(y \Rightarrow z)) \Rightarrow(y \Rightarrow((x \rightarrow u) \vee z))$

$[\mathrm{MP} \Rightarrow, 190,141]$

208. $((x \Rightarrow((y \rightarrow z) \vee u)) \Rightarrow v) \Rightarrow((y \rightarrow(x \Rightarrow u))$

$$
\Rightarrow v)
$$

$\left[\mathrm{MP}_{\Rightarrow}, 110,207\right]$

209. $(x \rightarrow(y \Rightarrow z)) \Rightarrow(y \Rightarrow(x \rightarrow z))$

$[\mathrm{MP} \Rightarrow, 208,184]$

210. $(x \Rightarrow(y \rightarrow(z \Rightarrow u))) \Rightarrow(x \Rightarrow(z \Rightarrow(y \rightarrow u)))$

$\left[\mathrm{MP}_{\rightarrow}, 68,209\right]$

211. $(x \rightarrow(y \Rightarrow z)) \rightarrow(y \Rightarrow(x \rightarrow z))$

$\left[\mathrm{MP}_{\rightarrow}, 8,209\right]$

212. $(x \rightarrow(y \Rightarrow z)) \rightarrow(y \rightarrow(x \rightarrow z))$

$\left[\mathrm{MP}_{\rightarrow}, 49,211\right]$

213. $((x \vee y) \Rightarrow(z \Rightarrow u)) \Rightarrow(z \Rightarrow(x \rightarrow u))$

214. $(x \vee(y \Rightarrow z)) \rightarrow(y \Rightarrow(x \vee z))$

$[\mathrm{MP} \Rightarrow, 210,166]$

$\left[\mathrm{MP}_{\rightarrow}, 103,55\right]$

$\left[\mathrm{MP}_{\rightarrow}, 73,214\right]$

215. $(x \rightarrow(y \vee(z \Rightarrow u))) \rightarrow(x \rightarrow(z \Rightarrow(y \vee u)))$

$\left[\mathrm{MP}_{\rightarrow}, 214,194\right]$

216. $(x \vee y) \Rightarrow((x \vee z) \vee y)$

$\left[\mathrm{MP}_{\Rightarrow}, 110,216\right]$

217. $(((x \vee y) \vee z) \Rightarrow u) \Rightarrow((x \vee z) \Rightarrow u)$

$[\mathrm{MP} \Rightarrow, 217,147]$

218. $(x \vee y) \Rightarrow(((x \vee z) \Rightarrow y) \Rightarrow y)$

$\left[\mathrm{MP}_{\rightarrow}, 128,218\right]$ 
220. $(x \Rightarrow(y \vee z)) \rightarrow((y \vee x) \Rightarrow(y \vee z)) \quad\left[\mathrm{MP}_{\rightarrow}, 8,219\right]$

221. $(x \Rightarrow(y \vee z)) \rightarrow(y \vee((y \vee x) \Rightarrow z)) \quad\left[\mathrm{MP}_{\rightarrow}, 189,220\right]$

222. $(x \rightarrow(y \Rightarrow(z \vee u))) \rightarrow(x \rightarrow(z \vee((z \vee y) \Rightarrow u)))\left[\mathrm{MP}_{\rightarrow}, 73,221\right]$

223. $(x \Rightarrow y) \rightarrow(z \vee((z \vee x) \Rightarrow y))$

$* 224$. $(x \Rightarrow y) \rightarrow((z \vee x) \Rightarrow(z \vee y))$

$\left[\mathrm{MP}_{\rightarrow}, 222,53\right]$

225. $(x \vee y) \Rightarrow(x \vee(z \vee y))$

$[\mathrm{MP} \rightarrow, 215,223]$

226. $(x \rightarrow(y \vee z)) \Rightarrow((x \vee z) \Rightarrow(y \vee z))$

$\left[\mathrm{MP}_{\rightarrow}, 224,10\right]$

227. $(x \rightarrow(y \rightarrow(z \vee u))) \Rightarrow(x \rightarrow((y \vee u) \Rightarrow(z$ $\vee u))$

$\left[\mathrm{MP}_{\rightarrow}, 154,225\right]$

*228. $(x \Rightarrow y) \rightarrow((x \vee z) \Rightarrow(y \vee z))$

229. $((x \Rightarrow(y \Rightarrow z)) \rightarrow(y \Rightarrow(z \wedge u)))$

$[\mathrm{MP} \Rightarrow, 160,226]$

$[\mathrm{MP} \Rightarrow, 227,102]$

$\Rightarrow((x \Rightarrow(y \Rightarrow z)) \Rightarrow(y \Rightarrow(z \wedge u))) \quad\left[\mathrm{MP}_{\Rightarrow}, 151,172\right]$

230. $(x \vee y) \Rightarrow((x \rightarrow(x \wedge y)) \Rightarrow y)$

$[\mathrm{MP} \Rightarrow, 205,183]$

231. $(x \rightarrow(x \wedge y)) \Rightarrow(x \rightarrow y)$

$[\mathrm{MP} \Rightarrow, 213,230]$

232. $(x \wedge y) \Rightarrow(x \rightarrow y)$

233. $((x \rightarrow y) \Rightarrow z) \rightarrow((x \wedge y) \Rightarrow z)$

$\left[\mathrm{MP}_{\rightarrow}, 97,231\right]$

$\left[\mathrm{MP}_{\rightarrow}, 84,232\right]$

234. $((x \rightarrow y) \Rightarrow z) \Rightarrow((x \wedge y) \Rightarrow z)$

$\left[\mathrm{MP}_{\Rightarrow}, 110,232\right]$

235. $(x \rightarrow((y \rightarrow z) \Rightarrow u)) \rightarrow(x \rightarrow((y \wedge z) \Rightarrow u))$

$\left[\mathrm{MP}_{\rightarrow}, 73,233\right]$

236. $(x \Rightarrow((y \rightarrow z) \Rightarrow u)) \Rightarrow(x \Rightarrow((y \wedge z) \Rightarrow u))$

$\left[\mathrm{MP}_{\rightarrow}, 68,234\right]$

237. $(x \Rightarrow y) \rightarrow((y \wedge z) \Rightarrow(x \rightarrow z))$

$\left[\mathrm{MP}_{\rightarrow}, 235,169\right]$

238. $(x \Rightarrow(y \rightarrow z)) \Rightarrow((y \wedge x) \Rightarrow(y \rightarrow z))$

$[\mathrm{MP} \Rightarrow, 236,152]$

239. $(x \Rightarrow(y \rightarrow z)) \Rightarrow((y \wedge x) \Rightarrow(y \wedge z))$

$\left[\mathrm{MP}_{\Rightarrow}, 203,238\right]$

240. $(x \Rightarrow(y \rightarrow z)) \rightarrow((y \wedge x) \Rightarrow(y \wedge z))$

$\left[\mathrm{MP}_{\rightarrow}, 8,239\right]$

$\left[\mathrm{MP}_{\rightarrow}, 94,240\right]$

*241. $(x \Rightarrow y) \rightarrow((z \wedge x) \Rightarrow(z \wedge y))$

242. $(x \Rightarrow y) \rightarrow(((y \wedge z) \Rightarrow x) \rightarrow((y \wedge z)$

$$
\Rightarrow(x \wedge z)))
$$

$\left[\mathrm{MP}_{\rightarrow}, 206,237\right]$

243. $(x \Rightarrow y) \rightarrow((y \Rightarrow((y \wedge z) \Rightarrow x)) \rightarrow((y \wedge z)$

$$
\Rightarrow(x \wedge z)))
$$

244. $((x \Rightarrow(y \Rightarrow z)) \rightarrow(y \Rightarrow(z \wedge u))) \rightarrow((x \Rightarrow$ $(y \Rightarrow z)) \Rightarrow(y \Rightarrow(z \wedge u)))$

245. $((x \Rightarrow(y \Rightarrow z)) \rightarrow(y \Rightarrow(z \wedge u)))$

$$
\rightarrow((x \Rightarrow z) \Rightarrow(y \Rightarrow(z \wedge u)))
$$

246. $(x \rightarrow((y \Rightarrow(z \Rightarrow u)) \rightarrow(z \Rightarrow(u \wedge v))))$

$\rightarrow(x \rightarrow((y \Rightarrow u) \Rightarrow(z \Rightarrow(u \wedge v))))$

$\left[\mathrm{MP}_{\rightarrow}, 159,242\right]$

$\left[\mathrm{MP}_{\rightarrow}, 8,229\right]$

$\left[\mathrm{MP}_{\rightarrow}, 106,244\right]$

$\left[\mathrm{MP}_{\rightarrow}, 73,245\right]$

247. $(x \Rightarrow y) \rightarrow((y \Rightarrow x) \Rightarrow((y \wedge z) \Rightarrow(x \wedge z))) \quad\left[\mathrm{MP}_{\rightarrow}, 246,243\right]$

${ }^{*} 248 .(x \Rightarrow y) \rightarrow((y \Rightarrow x) \rightarrow((x \wedge z) \Rightarrow(y \wedge z))) \quad\left[\mathrm{MP}_{\rightarrow}, 212,247\right]$ 


\section{References}

1. M. Baaz. Infinite-valued Gödel logic with 0-1-projections and relativisations. In Petr Hájek, editor, Gödel'96: Logical Foundations of Mathematics, Computer Science, and Physics, volume 6 of Lecture Notes in Logic, pages 23-33. Springer-Verlag, Brno, 1996.

2. R. J. Bignall. A non-commutative multiple-valued logic. In D. M. Miller, editor, Proceedings of the Twenty-first International Symposium on Multiple-Valued Logic, pages 49-54. IEEE Computer Society Press, Los Alamitos, 1991.

3. R. J. Bignall, M. Spinks, and R. Veroff. On the assertional logics of the generic pointed discriminator and generic pointed fixedpoint discriminator varieties. Manuscript, 2006.

4. W. J. Blok and D. Pigozzi. Algebraisable logics. Mem. Amer. Math. Soc., 77(396), 1989.

5. W. J. Blok and D. Pigozzi. Abstract algebraic logic and the deduction theorem. Bull. Symbolic Logic, To appear.

6. S. Burris and H. P. Sankappanavar. A Course in Universal Algebra. Number 78 in Graduate Texts in Mathematics. Springer-Verlag, New York, 1981.

7. J. Czelakowski and D. Pigozzi. Fregean logics. Ann. Pure Appl. Logic, 127:17$76,2004$.

8. J. M. Font and M. Rius. An abstract algebraic logic approach to tetravalent modal logics. J. Symbolic Logic, 65:481-518, 2000.

9. Petr Hájek. Metamathematics of Fuzzy Logic, volume 4 of Trends in Logic. Kluwer, Dordercht, 1998.

10. T. Hillenbrand et al. Waldmeister. http://www. waldmeister.org.

11. L. Humberstone. An intriguing logic with two implicational connectives. Notre Dame J. Formal Logic, 41:1-41, 2000.

12. L. Humberstone. Identical twins, deduction theorems and pattern functions: Exploring the implicative BCSK fragment of S5. J. Philosophical Logic, To appear.

13. I. Loureiro. Principal congruences of tetravalent modal algebras. Notre Dame J. Formal Logic, 26:76-80, 1985.

14. J. McCharen, R. Overbeek and L. Wos. Complexity and related enhancements for automated theorem-proving programs. Computers and Mathematics with Applications 2:1-16, 1976.

15. W. McCune. OTTER 3.0 Reference Manual and Guide, Technical Report ANL94/6, Argonne National Laboratory, Argonne, Illinois, 1994.

16. W. McCune, R. Padmanabhan, M. Rose and R. Veroff. Automated discovery of single axioms for ortholattices. Algebra Universalis, 52(4):541-549, 2005.

17. W. McCune. Prover9. http://www.mcs.anl.gov/〜mccune/prover9/.

18. H. Rasiowa. An Algebraic Approach to Non-Classical Logics. Number 78 in Studies in Logic and the Foundations of Mathematics. North-Holland Publ. Co., Amsterdam, 1974.

19. M. Spinks. A non-classical extension of classical implicative propositional logic. Bull. Symbolic Logic, 6:255, 2000. (Presented at the Austral. Assoc. Logic Annual Conference, Melbourne, July 1999.)

20. M. Spinks. On BCSK logic. Bull. Symbolic Logic, 9:264, 2003. (Presented at the Austral. Assoc. Logic Annual Conference, Canberra, Dec. 2002.)

21. R. Veroff. Using hints to increase the effectiveness of an automated reasoning program: case studies, J. Automated Reasoning 16(3):223-239, 1996. 
22. R. Veroff. Solving open questions and other challenge problems using proof sketches. J. Automated Reasoning 27(2):157-174, 2001.

23. R. Veroff. A shortest 2-basis for Boolean algebra in terms of the Sheffer stroke. Journal of Automated Reasoning 31(1):1-9, 2003.

24. V. Vychodil and I. Chajda. A note on residuated lattices with globalization. Int. J. Pure Appl. Math., To appear.

25. L. Wos. Milestones for automated reasoning with Otter. Int. J. on Artif. Intell. Tools 3-20, 2006. 
Robert Veroff

Computer Science Department

University of New Mexico

Albuquerque NM 87131

U.S.A.

veroff@cs.unm. edu

Matthew Spinks

Department of Philosophy

La Trobe University

Bundoora VIC 3086

Australia

mspinksau@yahoo.com.au 
fixed.tex; $14 / 04 / 2007 ; 11: 28 ;$ p.24 